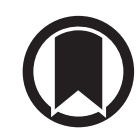

CrossMark

\section{Collapse induration of alveoli is an ultrastructural finding in a COVID-19 patient}

\author{
To the Editor:
}

The delicate alveolar blood-air barrier is a primary target in coronavirus disease 2019 (COVID-19). Its micro-architecture consists of an alveolar epithelium composed of type I and type II cells and covered with surfactant, a thin interstitium and a capillary endothelium. Of particular relevance for the pathogenesis of severe COVID-19 is the infection of type II alveolar epithelial cells [1]. Based on their dual function as producers of surfactant and as precursors for both epithelial cell types, surfactant alterations and aberrant epithelial regeneration can be expected.

Although there has been some discussion whether the pathophysiology of COVID-19 is a typical form of acute respiratory distress syndrome (ARDS) or a distinct form of lung injury, diffuse alveolar damage (DAD) as the histopathological correlate of acute lung injury and ARDS is a regular finding in COVID-19 [2-4]. DAD includes an early exudative (or acute) phase with alveolar epithelial injury, oedema formation and surfactant alterations, and a late proliferative (or organising) phase with cuboidal metaplasia of type II alveolar epithelial cells and thickening of inter-alveolar septa, finally resulting either in restoration or in fibrosis [5]. In the context of DAD, the concept of collapse (or atelectatic) induration as an important event in the progression of lung injury towards fibrosis has been emphasised [6-8]. An initial alveolar epithelial injury with surfactant alterations leads to alveolar instability and collapse, which becomes irreversible due to "glueing" of infolded and denuded alveolar epithelial basal laminae and re-epithelialisation over infolded alveoli.

Morphological evidence for collapse induration, in particular the tracing of the thin alveolar epithelial basal lamina, requires a resolution that can only be provided by transmission electron microscopy (TEM) [8]. Here, we report alterations at the TEM level demonstrating collapse induration in post mortem samples from a COVID-19 patient. The 62-year-old male patient tested positive for SARS-CoV-2, was admitted to hospital due to new onset of dyspnoea and fever, and intubated 1 day later. He received catecholamines, antibiotics, renal replacement therapy but no anti-viral or anti-inflammatory therapy. Under lung protective mechanical ventilation (positive end-expiratory pressure 16-21 mbar; tidal volume $\sim 6 \mathrm{~mL}$ per kg bodyweight; arterial oxygen tension/inspired oxygen fraction 120-150) and intermittent prone positioning, lung infiltrates partially resolved. After 13 days of mechanical ventilation, the patient deceased due to sudden onset septic shock.

Autopsy was performed on the legal basis of $\$ 1$ SRegG BE of the autopsy act of Berlin and $\$ 25(4)$ of the German Infection Protection Act. This study was approved by the ethics committee of the Charité Universitätsmedizin Berlin (EA 1/144/927 13) and was in compliance with the Declaration of Helsinki.

To inactivate potential infectivity of the virus, two larger tissue samples from the right lower lobe were fixed with 3\% formaldehyde (prepared from freshly depolymerised paraformaldehyde) in $0.1 \mathrm{M}$ cacodylate buffer for $12 \mathrm{~h}$ followed by changing the fixative for another $12 \mathrm{~h}$ (following the recommendations by the Robert Koch Institute, Berlin, Germany). For TEM, 26 smaller tissue samples were cut and fixed with $1.5 \%$ glutaraldehyde/1.5\% paraformaldehyde in $0.15 \mathrm{M}$ Hepes buffer overnight and post-fixed with $1 \%$ $\mathrm{OsO}_{4}$ for $1 \mathrm{~h}$ followed by $0.1 \%$ tannic acid for $0.5 \mathrm{~h}$ and washing steps with $1 \%$ sodium sulfate (all in $0.05 \mathrm{M}$ Hepes). Samples were finally dehydrated in ethanol, infiltrated with propylene oxide and

@ERSpublications

Electron microscopy reveals collapse induration with alveolar epithelial cell death, basal lamina denudation, collapse and sealing of alveoli in a COVID-19 patient, implicating surfactant dysfunction and alveolar instability in fibrosis initiation https://bit.ly/38yEX2g

Cite this article as: Ochs M, Timm S, Elezkurtaj S, et al. Collapse induration of alveoli is an ultrastructural finding in a COVID-19 patient. Eur Respir J 2021; 57: 2004165 [https://doi.org/10.1183/ 13993003.04165-2020]. 


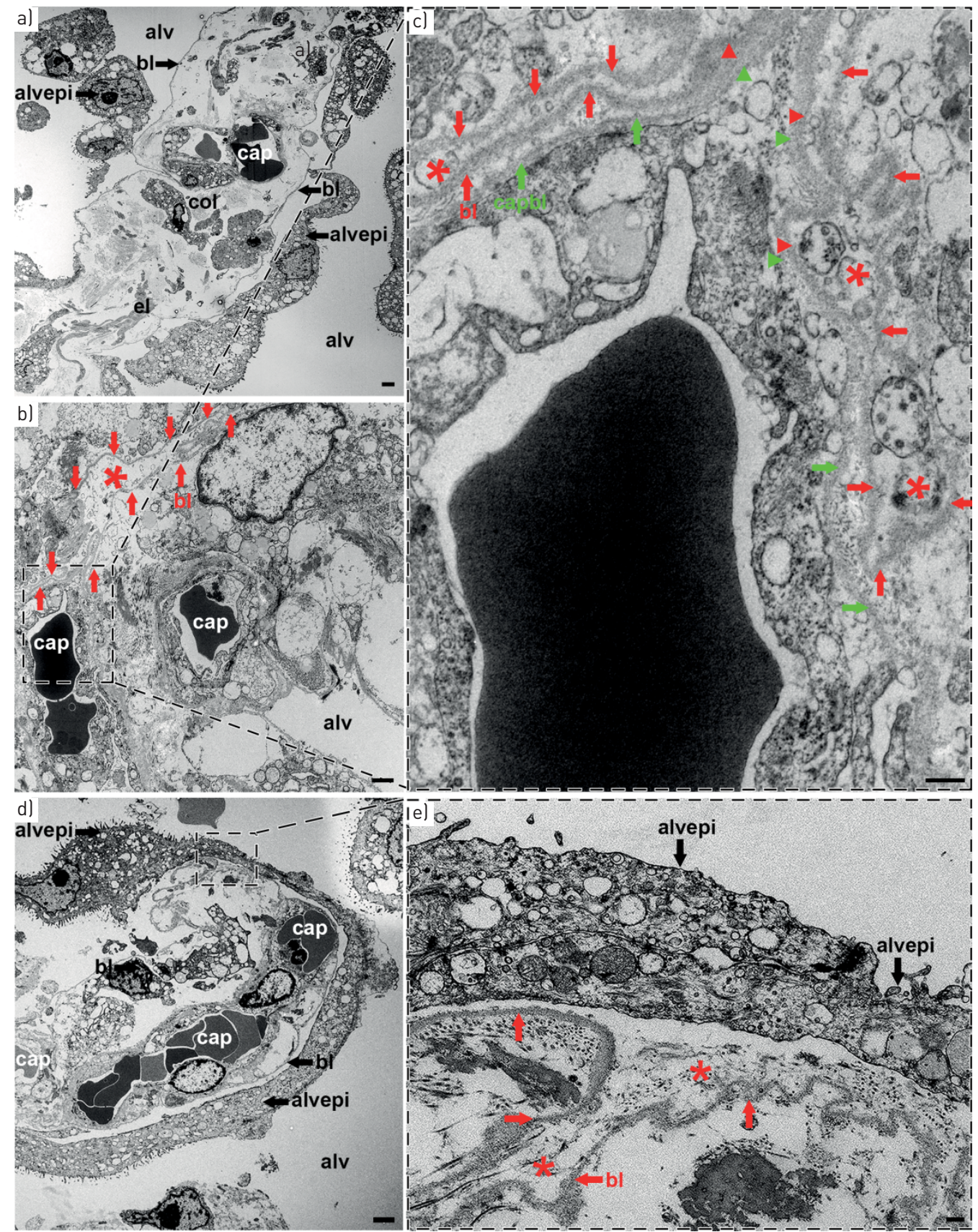

FIGURE 1 a) Inter-alveolar septum containing capillaries (cap) and interstitium with collagen fibrils (col) and elastic fibres (el). The septum is thickened due to interstitial oedema. The alveolar epithelium (alvepi) on both sides of the septum is partially detached towards the alveolar lumen (alv). At the sites of detachment, the alveolar epithelial basal lamina (bl) is denuded. Scale bar: $2 \mu \mathrm{m}$. b) Inter-alveolar septum with thickened blood-air barrier between air in the alveolar lumen (alv) and blood in the capillaries (cap). Note the infolding of the denuded alveolar epithelial basal lamina (bl; red arrows) with collapsed alveolar lumen (red asterisk). Scale bar: $1 \mu \mathrm{m}$. c) Higher magnification of the boxed area (in b) showing the infolded alveolar epithelial (bl; red arrows) as well as the capillary endothelial basal lamina (capbl; green arrows), which are partly fused (red and green arrowheads). The capillary endothelial basal lamina continues to surround the capillary endothelium whereas the alveolar epithelial basal lamina turns back towards the origin of the infolding, thus bordering a collapsed alveolar lumen (red asterisks). Scale bar: $250 \mathrm{~nm}$. d) Inter-alveolar septum with capillaries (cap). The alveolar epithelium (alvepi) shows thick cell extensions with microvilli, a characteristic of transitional cells between a type II and a type I alveolar epithelial cell phenotype, thus indicating epithelial cell proliferation. alv: alveolar lumen; bl: alveolar epithelial basal lamina. Scale bar: $2.5 \mu \mathrm{m}$. e) Higher magnification of the boxed area (in d). Entrance into an alveolar infolding with collapsed alveolar lumen (red asterisks) bordered by a denuded alveolar epithelial basal lamina (bl; red arrows). Note the "sealing" of the entrance by two overgrowing alveolar epithelial cells (alvepi). Scale bar: $250 \mathrm{~nm}$. 
embedded in Epon. Ultrathin sections (about $70 \mathrm{~nm}$ ) of five different TEM blocks were counterstained with lead citrate and examined with a Leo 906 TEM (Zeiss, Oberkochen, Germany).

Ultrastructural findings are illustrated in figure 1. Alveolar septa were thickened due to the formation of interstitial oedema. The alveolar epithelium had an increased coverage by type II cells, indicating hyperplasia as an attempt to repair areas of epithelial injury. In places, type II cells were seen in transition towards a type I cell phenotype. Alveolar epithelial cells were partly swollen or necrotic, with isolated cells or connected cell layers desquamated into the alveolar lumen. As a consequence, the alveolar epithelial basal lamina was to a large extent denuded. In all of the five TEM blocks studied, infoldings of denuded basal laminae could be observed. These infoldings bordered collapsed alveolar lumina. At their entrance, these infoldings were partly covered with proliferating alveolar epithelial cells.

The post mortem ultrastructural findings from a COVID-19 patient presented here are similar to previous descriptions in DAD [6] and idiopathic bronchiolitis obliterans-organising pneumonia [9], as well as to findings in the bleomycin injury model in rats and in explants from patients with idiopathic pulmonary fibrosis that underwent lung transplantation $[8,10]$, thus indicating that they are a common reaction pattern to alveolar epithelial injury. The bleomycin model is of particular value in the context of COVID-19, because it induces a fibrotic response following acute lung injury, which can be followed in a pursuable, reproducible and revisable manner [11]. The findings can be interpreted as a sequence of events: damage of alveolar epithelial cells leads to surfactant dysfunction and alveolar instability with subsequent micro-atelectasis. The denuded basal laminae of collapsed alveoli coalesce, and the entrances into collapsed alveoli are sealed by proliferating alveolar epithelial cells, thus forming thickened septa. The consequences are a loss of alveolar airspace volume and a loss of alveolar gas exchange surface.

The ultrastructural alterations found in this post mortem patient sample provide morphological evidence for a possible transition into lung fibrosis after COVID-19 lung injury. Although the mechanical ventilation of the patient may have contributed to the progression of DAD [12], acute DAD is also seen in COVID-19 pneumonia independent of mechanical ventilation [2, 4]. Without the development of septic shock this patient might have survived but developed fibrosis. In the bleomycin animal model of lung injury and fibrosis, this transition towards lung fibrosis could be attenuated by exogeneous surfactant therapy [13]. This suggests that restoration of the surfactant system, e.g. by exogeneous surfactant therapy, might be considered as an early therapeutic intervention for COVID-19 patients. A similar concept has also been put forward by others $[14,15]$ and is in line with the general idea of preventive anti-fibrotic therapy in COVID-19 [11].

In summary, post mortem ultrastructural findings in a COVID-19 patient show alveolar epithelial cell death, denudation of the alveolar epithelial basal lamina, alveolar collapse and collapse induration. These alterations indicate early surfactant dysfunction as an important event in the pathophysiology of COVID-19.

Matthias Ochs $\oplus^{1,2,3}$, Sara Timm $^{3}$, Sefer Elezkurtaj ${ }^{4}$, David Horst ${ }^{4}$, Jenny Meinhardt $\oplus^{5}$, Frank L. Heppner ${ }^{5,6,7,8}$, Steffen Weber-Carstens $\oplus^{9}$, Andreas C. Hocke ${ }^{2,10}$ and Martin Witzenrath ${ }^{2,10}$

${ }^{1}$ Institute of Functional Anatomy, Charité - Universitätsmedizin Berlin, corporate member of Freie Universität Berlin and Humboldt-Universität zu Berlin, Berlin, Germany. ${ }^{2}$ German Center for Lung Research (DZL), Berlin, Germany. ${ }^{3}$ Core Facility Electron Microscopy, Charité - Universitätsmedizin Berlin, corporate member of Freie Universität Berlin and Humboldt-Universität zu Berlin, Berlin, Germany. ${ }^{4}$ Dept of Pathology, Charité - Universitätsmedizin Berlin, corporate member of Freie Universität Berlin and Humboldt-Universität zu Berlin, Berlin, Germany. ${ }^{5}$ Dept of Neuropathology, Charité - Universitätsmedizin Berlin, corporate member of Freie Universität Berlin and HumboldtUniversität zu Berlin, Berlin, Germany. ${ }^{6}$ Berlin Institute of Health (BIH), Berlin, Germany. ${ }^{7}$ Cluster of Excellence, NeuroCure, Berlin, Germany. ${ }^{8}$ German Center for Neurodegenerative Diseases (DZNE) Berlin, Berlin, Germany. ${ }^{9}$ Dept of Anesthesiology and Operative Intensive Care Medicine, Charité - Universitätsmedizin Berlin, corporate member of Freie Universität Berlin and Humboldt-Universität zu Berlin, Berlin, Germany. ${ }^{10}$ Dept of Infectious Diseases and Respiratory Medicine, Division of Pulmonary Inflammation, Charité - Universitätsmedizin Berlin, corporate member of Freie Universität Berlin and Humboldt-Universität zu Berlin, Berlin, Germany.

Correspondence: Matthias Ochs, Institute of Functional Anatomy, Charité - Universitätsmedizin Berlin, Philippstr. 11, 10115 Berlin, Germany. E-mail: matthias.ochs@charite.de

Received: 12 Nov 2020 | Accepted: 23 Dec 2020

Acknowledgements: The expert technical assistance by Petra Schrade (Core Facility for Electron Microscopy, Charité) is highly acknowledged.

Author contributions: Conception and design: M. Ochs and M. Witzenrath. Gathering of materials and data: S. Elezkurtaj, D. Horst, J. Meinhardt, F.L. Heppner, S. Weber-Carstens, A.C. Hocke and M. Witzenrath. Acquisition, analysis and interpretation of data: M. Ochs and S. Timm. Drafting and/or revising of manuscript: M. Ochs, S. Timm and M. Witzenrath. Final approval of manuscript: M. Ochs, S. Timm, S. Elezkurtaj, D. Horst, J. Meinhardt, F.L. Heppner, S. Weber-Carstens, A.C. Hocke and M. Witzenrath. 
Conflict of interest: M. Ochs has nothing to disclose. S. Timm has nothing to disclose. S. Elezkurtaj has nothing to disclose. D. Horst has nothing to disclose. J. Meinhardt has nothing to disclose. F.L. Heppner has nothing to disclose. S. Weber-Carstens has nothing to disclose. A.C. Hocke has nothing to disclose. M. Witzenrath reports grants from Actelion, Noxxon and Quark Pharma, grants and personal fees from Bayer Health Care, Biotest, Boehringer Ingelheim, Pantherna and Vaxxilon, personal fees from Actelion, Aptarion, AstraZeneca, Berlin Chemie, Chiesi, GlaxoSmithKline and Novartis, outside the submitted work.

Support statement: M. Ochs was supported by the German Ministry of Education and Research (BMBF: 01DG14009) and by the Berlin University Alliance (BUA: 501_Elektronenmikroskopie). A.C. Hocke was supported by the Berlin University Alliance (BUA) GC2 Global Health (Corona Virus Pre-Exploration Project), by the German Ministry of Education and Research (BMBF: RAPID, Organo-Strat, alvBarriere-COVID-19), by the German Research Foundation (DFG: SFB-TR84; B6, Z1a), and by the Berlin Institute of Health (BIH), Charité 3R, and Charité-Zeiss MultiDim. M. Witzenrath was supported by grants from the German Research Foundation (DFG: SFB-TR84 C6 and C9) and by the German Ministry of Education and Research (BMBF: CAPSyS (01ZX1304B), SYMPATH (01ZX1906A), and PROVID (01KI20160A)). Funding information for this article has been deposited with the Crossref Funder Registry.

\section{References}

$1 \quad$ Mason RJ. Pathogenesis of COVID-19 from a cell biology perspective. Eur Respir J 2020; 55: 2000607.

2 Bradley BT, Maioli H, Johnston R, et al. Histopathology and ultrastructural findings of fatal COVID-19 infections in Washington state: a case series. Lancet 2020; 396: 320-332.

3 Carsana L, Sonzogni A, Nasr A, et al. Pulmonary post-mortem findings in a series of COVID-19 cases from northern Italy: a two-centre descriptive study. Lancet Infect Dis 2020; 20: 1135-1140.

4 Konopka KE, Nguyen T, Jentzen JM, et al. Diffuse alveolar damage (DAD) resulting from coronavirus disease 2019 infection is morphologically indistinguishable from other causes of DAD. Histopathology 2020; 77: 570-578.

5 Katzenstein AL, Bloor CM, Leibow AA. Diffuse alveolar damage - the role of oxygen, shock, and related factors. Am J Pathol 1976; 85: 209-228.

6 Katzenstein AL. Pathogenesis of "fibrosis" in interstitial pneumonia: an electron microscopic study. Hum Pathol 1985; 16: 1015-1024.

7 Todd NW, Atamas SP, Luzina IG, et al. Permanent alveolar collapse is the predominant mechanism in idiopathic pulmonary fibrosis. Expert Rev Respir Med 2015; 9: 411-418.

8 Ochs M, Knudsen L, Hegermann J, et al. Using electron microscopes to look into the lung. Histochem Cell Biol 2016; 146: 695-707.

9 Myers JL, Katzenstein AL. Ultrastructural evidence of alveolar epithelial injury in idiopathic bronchiolitis obliterans-organizing pneumonia. Am J Pathol 1988; 132: 102-109.

10 Lutz D, Gazdhar A, Lopez-Rodriguez E, et al. Alveolar derecruitment and collapse induration as crucial mechanisms in lung injury and fibrosis. Am J Respir Cell Mol Biol 2015; 52: 232-243.

11 George PM, Wells AU, Jenkins RG. Pulmonary fibrosis and COVID-19: the potential role for antifibrotic therapy. Lancet Respir Med 2020; 8: 807-815.

12 Albert RK. The role of ventilation-induced surfactant dysfunction and atelectasis in causing acute respiratory distress syndrome. Am J Respir Crit Care Med 2012; 185: 702-708.

13 Steffen L, Ruppert C, Hoymann HG, et al. Surfactant replacement therapy reduces acute lung injury and collapse induration related lung remodeling in the bleomycin model. Am J Physiol Lung Cell Mol Physiol 2017; 313: L313-L327.

14 Busani S, Dall'Ara L, Tonelli R, et al. Surfactant replacement might help recovery of low-compliance lung in severe COVID-19 pneumonia. Ther Adv Respir Dis 2020; 14: 1753466620951043.

15 Koumbourlis AC, Motoyama EK. Lung mechanics in COVID-19 resemble respiratory distress syndrome, not acute respiratory disctress syndrome: could surfactant be a treatment? Am J Respir Crit Care Med 2020; 202: 624-626.

Copyright $\odot$ The authors 2021. 